- Ninety per cent of GDPs in this survey treated patients on warfarin.

- There is a wide variation in the management of patients on warfarin in primary dental care.

- Guidelines for the management of patients on warfarin in primary care have been produced, but the concerns of GDPs in this area need to be addressed by prospective audits in this setting.

\title{
An assessment of the management of patients on warfarin by general dental practitioners in South West Wales
}

\author{
A. Muthukrishnan ${ }^{1}$ and K. Bishop ${ }^{2}$
}

\begin{abstract}
For anticoagulation therapy, warfarin is used to reduce the risk of thrombo-embolic events in patients with mechanical prosthetic heart valves, certain cardiovascular conditions, deep vein thrombosis (DVT) and hypercoagulable states. ${ }^{1}$ The International Normalised Ratio (INR) is used as a measure of anticoagulation and is expressed as a ratio of the patient's prothrombin time (PT) to control prothrombin time. In a person with a normal PT, the INR is approximately 1 . The therapeutic range is the value of INR or degree of anticoagulation required to prevent the development of serious thrombo-embolism and is normally maintained between 2.0 and 4.5. It is important to recognize that the INR is only valid for patients on well controlled anticoagulant therapy, ie where the level of anticoagulation is reasonably stable over a moderate length of time. ${ }^{2}$
\end{abstract}

\section{DENTAL IMPLICATIONS OF TREATING AN ANTICOAGULATED PATIENT}

These include:

1. The risk of uncontrolled bleeding from the dental procedure in the absence of appropriate management.

2. The risk of a thromboembolic event related to the interruption of anticoagulation therapy.

3. The management of the underlying cause for which anticoagulant therapy has been instituted.

Studies relating to the management of anticoagulation in patients on warfarin have been based on dental extractions carried out in hospital settings. ${ }^{3-7}$ There is no published evidence about the management of patients on warfarin undergoing dental treatment in primary care by the General

${ }^{1}$ Associate Specialist in Restorative Dentistry, Morriston Hospital, Swansea SA6 6NL; ${ }^{2}$ Consultant in Restorative Dentistry and Implantology, Morriston Hospital, Swansea SA6 6NL

Correspondence to: Akhila Muthukrishnan

E-mail: akhila.muthukrishnan@swansea-tr.wales.nhs.uk
Dental Services in the United Kingdom. Additional factors which may influence the management of such patients in primary care include access to hospital resources, and constraints specific to the General Dental Services in the UK that have been reported anecdotally. ${ }^{8,9}$

\section{THE INR DILEMMA}

The guidelines in the Dental Practitioners Formulary regarding the management of patients on long-term anticoagulant therapy advice consultation with the patient's medical practitioner and adjustment of the INR to approximately 2.0. ${ }^{10}$

Other guidelines previously recommended that minor surgery (simple extractions of two or more teeth) could be safely carried out up to an INR of 2.5 times normal. ${ }^{2}$ These recommendations were later changed to an INR of 3.5 times normal. ${ }^{11}$

The Northwest Medicines Information Centre recently introduced guidelines which recommend that patients on warfarin can have dental surgical procedures carried out in primary care up to an INR of 4.0. ${ }^{12}$

\section{OBJECTIVE}

The objective of this study was to assess the management of patients on warfarin by primary care dentists in South West Wales and to evaluate factors which might affect this practice. In addition, the regimes followed by these clinicians were compared with the current guidelines.

\section{QUESTIONNAIRE BASED SURVEY AND RESPONSES}

A standard questionnaire was sent to 250 dentists on the South-West Wales dental post-graduate mailing list. This mailing list included all dentists in the region working in the General Dental Service (GDS), Community Dental Service (CDS) and Hospital Dental Service (HDS). The clinicians were requested to complete the questionnaire anonymously and to return them in a supplied stamped addressed envelope. The following information was obtained.

\section{Data collected}

One hundred and sixty completed questionnaires were received (response rate of 64\%). Among the responses, 155 (97\%) were from GDPs, 4 (2.5\%) from the HDS, and $1(0.5 \%)$ from the CDS. The numbers from HDS and CDS were insufficient to obtain any meaningful results, and were therefore omitted from any subsequent analysis. A reply rate of $64 \%$ of which $97 \%$ 


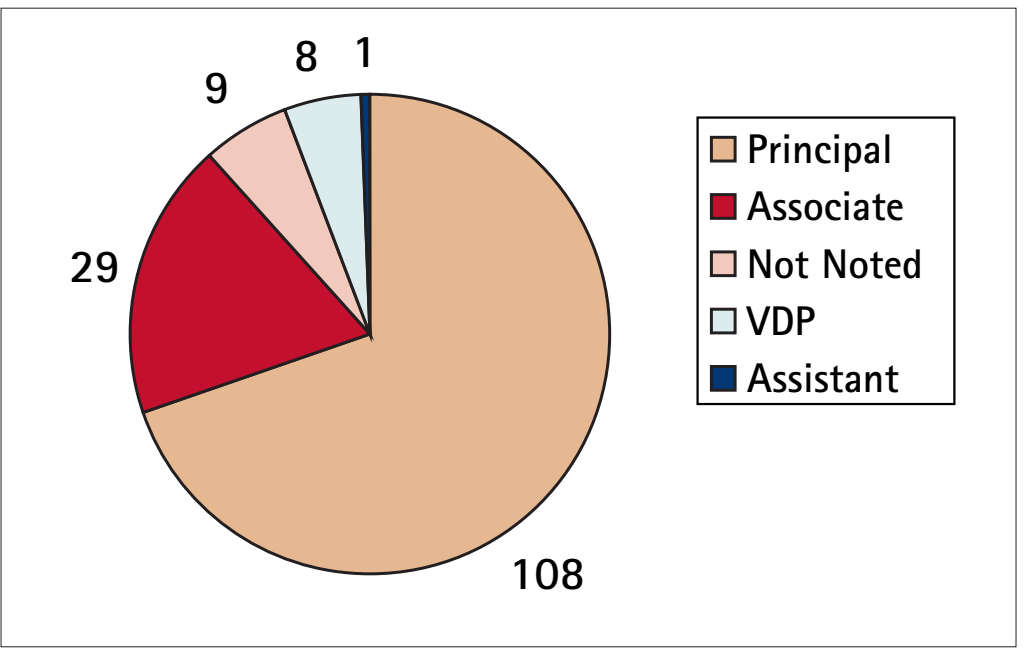

Fig. 1 Position of GDP in the practice

were GDPs means that restricting the analysis to this group of clinicians resulted in a good representation of the views and practices of GDPs in the area.

\section{GDP's experience and position in practice} The majority of GDPs (108) were principals. Fifty-eight (37\%) GDPs had worked in the HDS in the past. Neither of these two factors had any apparent effect on the management of warfarinised patients. On the assumption that a principal is more experienced than a VDP, it would appear that GDPs in this study had a similar view on the management of warfarinised patients irrespective of their level of experience. The finding that previous hospital experience did not affect the management of warfarinised patients would suggest that similar practices were followed in hospitals and primary care. Alternatively, they may have failed to implement past practices into their current working environment.

\section{Non-treatment}

Of the 155 GDPs who responded, 9\% did not treat any patient taking warfarin for a variety of reasons. The explanations for their actions if provided were recorded and included 'time consuming', 'difficulty in co-ordinating INR prior to treatment', 'no support from GP/cardiologist,' and 'potential medico-legal problems.'

It has been suggested that the main problem in performing extractions in general dental practice is likely to be financial. ${ }^{13}$ It would appear that GDPs perceive other barriers to the care of this group of patients. These need to be addressed in addition to the financial issue.

\section{Opinion of procedure related risk of bleeding}

Clinicians were asked to rate the potential risk of haemorrhage associated with a number of routine dental procedures as either 'high' or 'low'. For this purpose, a list of procedures was provided including local infiltration, nerve blocks, scaling and subgingival debridement. Evidence related to the risk of bleeding in patients on warfarin has been mainly based on dental extractions, alveolar surgery and gingival surgery. ${ }^{3-7}$ Some authors have reported that there was no increased risk of bleeding associated with routine dental procedures such as scaling and restorations. However, no treatment was carried out on these patients if their INR was above 2.5.14 Dental extraction was therefore cited as an example of a procedure with a 'high' potential risk of bleeding. The assessment of procedure related risk by GDPs is listed in Table 1.

\section{Local anaesthetic nerve blocks}

Opinion was divided regarding the bleeding risk associated with an inferior dental block. Forty-eight per cent considered it as 'high risk' while $41 \%$ regarded it as 'low'. This continues to be a contentious issue among dentists. One study specifically mentions the safe use of inferior alveolar, lingual and mental nerve blocks in a series of 133 extractions, though there is no mention of the number of nerve blocks administered. ${ }^{3}$ In another significant randomised controlled trial, dental extractions were carried out in 109 patients. Regional blocks were used in the mandible, though again, there is no mention of the number. ${ }^{6}$ Older studies have reported ecchymoses, hematomas or facial swellings as post-operative complications as a result of nerve blocks in anticoagulated patients undergoing extractions. ${ }^{15,16}$ However, these complications could have resulted from the surgical procedure itself. None of these authors gave specific information about the exact local anaesthetic methodology, ie exact sites of injections, the number of injections given, the volume of anaesthetic used, the rate of infusion, the size of the needle used and the presence or absence of negative aspirate. ${ }^{17}$

Current guidelines suggest that regional blocks are avoided whenever possible but where unavoidable, administered with an aspirating syringe. ${ }^{12}$ The authors support this view in practice. However, aspiration is mandatory in all nerve blocks and still does not affect the possibility of bleeding from needle insertion. An atraumatic injection technique is probably more important.

\section{Periodontal procedures}

Periodontal examinations were considered 'low risk' (84\%) as was supragingival scaling $(90 \%)$. However $71 \%$ of GDPs considered subgingival debridement (SGD) as 'high risk'. There is justifiable concern in this regard as SGD in anticoagulated patients may cause significant bleeding from inflamed tissue at multiple sites which can be considered a different clinical challenge from a simple extraction or localised surgical procedure.

There is clearly a need for evidence specific to SGD in anticoagulated patients. Until such evidence is available, the authors suggest that good plaque control is first established and the mouth is treated in quadrants if necessary to reduce bleeding.

All other procedures were rated as relatively low risk in terms of bleeding.

It would appear that the other dental procedures listed in Table 1 can be safely carried out with the use of local haemostatic procedures only.

\begin{tabular}{|c|c|c|c|}
\hline Procedure & High & Low & Not noted \\
\hline Sub-gingival debridement & $110(71 \%)$ & $32(20 \%)$ & $13(9 \%)$ \\
\hline Supra-gingival scaling & $7(4.5 \%)$ & 140 (90\%) & $8(5 \%)$ \\
\hline Inferior dental block & $74(48 \%)$ & $64(41 \%)$ & $17(11 \%)$ \\
\hline Infiltration & $8(5 \%)$ & $133(86 \%)$ & $14(9 \%)$ \\
\hline Sub-gingival restorations & 49 (31.6\%) & $93(60 \%)$ & $13(8.4 \%)$ \\
\hline Periodontal examination & $14(9 \%)$ & 131 (84\%) & $10(6.6 \%)$ \\
\hline \multicolumn{2}{|c|}{ Pulp extirpation and RCT preparation20 (12\%) } & $125(81 \%)$ & $10(6.4 \%)$ \\
\hline RCT obturation & $5(3 \%)$ & 141 (91\%) & $9(6 \%)$ \\
\hline
\end{tabular}




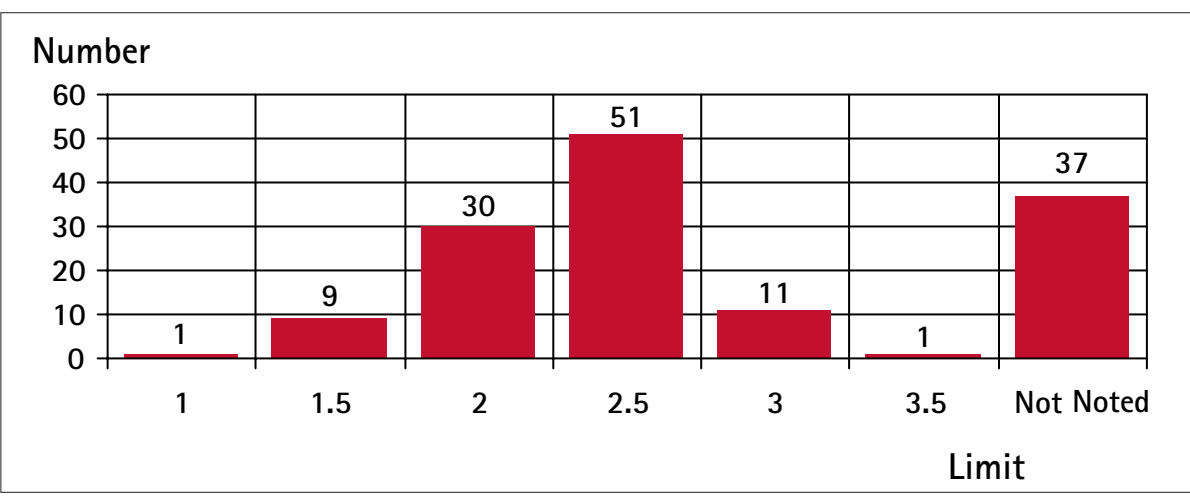

Fig. 2 Upper limit of INR considered acceptable for treatment

\section{Management of anticoagulation}

Clinicians who treated warfarinised patients were asked about the following questions in relation to procedures that they felt had a 'high' risk of bleeding.

1. Whether they advised patients to stop or reduce the warfarin dose

Twenty-two (15\%) of clinicians advised patients to either stop or reduce their dose of warfarin for 1 to 3 days prior to dental treatment without seeking a medical opinion. However, 13 (9\%) added that they were selective in the treatment they provided and referred patients to hospital for extractions and minor dento-alveolar surgery.

It would appear that GDPs followed recommendations from the Dental Practitioner's formulary with regards to obtaining an INR around 2.0. However, this guidance note clearly advises that the patient's medical practitioner is consulted in this regard. Guidelines from the Northwest Medicines Information Centre were not available to the GDPs surveyed, but may have been accessed by them since then through local hospital departments.

2. Whether they sought advice from the patient's general medical practitioner (GMP) or cardiologist

Fifty-eight (41\%) of GDPs sought advice from the general medical practitioner and $37(26 \%)$ from the cardiologist regarding the management of the anticoagulant regime. Forty-five (32\%) appeared to seek advice from both or either.

\section{INR assessment prior to treatment}

Seventy-seven (55\%) of GDPs checked INR prior to treatment, $14(10 \%)$ of replies provided no information. The questionnaire itself was not specific regarding the exact timing of INR assessment before treatment. The issue of the timing of pre-operative INR is not specified in the advice in relation to oral anticoagulants in the Dental Practitioners' Formulary.

Other guidelines advise that an INR is measured within 24 hours of the proposed procedure. ${ }^{12}$ This is necessary because warfarin control can be affected by obvious factors such as drug interactions, intercurrent illness or liver disease. It can also be affected by major changes in diet (especially involving salad and vegetables) and in alcohol consumption. ${ }^{18}$ As a result, dental treatment needs to be provided within 24 hours of the patient's regular INR assessment or an additional evaluation carried out.

4. What they considered to be the safe upper limit of INR for carrying out 'high risk of bleeding' procedures

The upper limit of INR considered to be 'safe' by GDPs is charted in Figure 2 and ranged from 1 to 3.5. Fifty-one (36\%) considered 2.5 as the upper limit, while $40(28 \%)$ considered it to be in a range between 1 and 2. GDPs would appear to have followed the guidelines available to them at the time. ${ }^{10}$ This continued to be the advice to GDPs in 2002 and is at variance with guidelines published by the Northwest Medicines Information Centre in 2001.

The latter suggests that the anticoagulation regime does not require alteration if the INR is $\leq 4.0$ for minor surgical procedures, eg simple extraction of up to three teeth, gingival surgery and the surgical removal of teeth. It is also recommended that patients should be referred to a hospital based clinic for the management of a high risk procedure if they are maintained with an INR $>4.0$, the INR is poorly controlled, or there are other concurrent medical problems that complicate management.

\section{Are our medical colleagues aware of current guidelines?}

Since the majority of GDPs in this study perceive the 'safe limit' of an INR to be 2.5 (see later), it is possible that they may have requested the GMP/cardiologist to lower the warfarin dose rather than initiate the changes themselves. From this study it is unclear whether such requests were carried out. However, it is reasonable to assume that their views were reinforced by the subsequent action of medical colleagues.

A 1996 survey of physicians in the USA found that more than $70 \%$ of respondents recommended interrupting continuous anticoagulant therapy for at least some dental procedures. However many physicians do not understand dental procedures. In the above survey, physicians more often recommended withdrawing anticoagulant therapy for patients about to undergo endodontic therapy than they do for patients about to undergo scaling. ${ }^{19}$ Guidelines relating to anticoagulation were published in $1998 .^{20,21}$ It is possible, therefore, that many general medical practitioners themselves are unaware of the current recommendations. This could be the basis of a further survey.

5. Their potential management of the anticoagulation regime in relation to the following conditions for which warfarin therapy is commonly prescribed; deep vein thrombosis (DVT), pulmonary embolism $(P E)$, atrial fibrillation (AF), valvular disease and a prosthetic valve.

In all responses, the method of managing the anticoagulant regime selected by GDPs remained unchanged irrespective of the underlying cause for which anticoagulant therapy had been instituted. This is of some concern, since a total of $64 \%$ of GDPs aimed for a target INR less than 2.5. However, the target therapeutic range for these conditions is 3.5. ${ }^{22,23}$ Therefore any reduction of INR in this group would predispose them to a higher risk of serious thromboembolism.

Conditions which may lead to the use of anticoagulation therapy may be divided into 'low' and 'high' risk based on the associated risk of thromboembolic events. $^{24}$ High-risk conditions are mechanical mitral valves, atrial fibrillation (AF) associated with valvular heart disease, including the presence of a mechanical valve, and mechanical valves in patients who have suffered a prior thromboembolic event. ${ }^{25,26}$

If the anticoagulant state requires adjustment when any of the above conditions exist, for example, before major surgery, then heparin therapy is indicated. ${ }^{27-29}$ However, this should not be carried out in primary care.

Increases in the aged population and medical advances have resulted in a greater need for the management of this patient group. An additional problem with dental procedures such as scaling is their relatively high frequency in comparison with minor oral surgical procedures. The importance of regular dental check-ups with 
Table 2 Summary of guidelines for surgical management and dental treatment of patients on warfarin in primary care c $^{*}$

General measures

1. Check need for endocarditis prophylaxis and treat appropriately

2. Be aware of the high risk of thromboembolism associated with prosthetic valves

3. Schedule appointment within 24 hours of INR measurement

4. Proceed with treatment ${ }^{\dagger}$ if $\mid N R<1=4.0^{\ddagger}$

5. Use local measures to control bleeding with appropriate post-operative advice

6. Be wary of drug interactions with warfarin and advise patients accordingly

*Patients with concomitant liver disease, renal failure, other bleeding disorders and those currently receiving cytotoxic medication or radiotherapy should be referred to hospital

${ }^{\dagger}$ All dental procedures listed in Table 1 and minor surgical procedures such as simple extraction of up to three teeth, localised gingival surgery and the surgical removal of teeth are suitable for treatment in primary care.

${ }^{\ddagger}$ If the INR is $>4.0$, there must be consultation with the clinician responsible for their anticoagulation. If the patient is maintained $>4.0$ or has very erratic control, they must be referred to hospital

Table 3 Summary of guidelines for surgical management and dental treatment of patients on warfarin in primary care ${ }^{12}$

\section{Local measures}

1. Local anaesthetic containing vasoconstrictor should be administered by infiltration or by intraligamentary injection wherever practical. If there is no alternative to regional nerve blocks, it should be administered cautiously with an aspirating syringe

2. After extraction, sockets should be gently packed with an absorbable haemostatic dressing eg oxidised cellulose (Surgice $\left.{ }^{\circledR}\right)$, then carefully sutured. Pressure should be applied by using a gauze pad that the patient bites down on for 15 to 30 minutes.

In the post-operative period, the patient should be advised:

- Look after the initial clot by resting while the local anaesthetic wears off and the clot forms (2-3 hours)

- To avoid rinsing the mouth for 24 hours

- Not to suck hard or stick the tongue or any foreign object into the socket

- To avoid hot liquids and hard foods for the rest of the day

- Not to chew on the affected side for 3 days

- If bleeding continues or restarts to apply pressure over the socket using a folded clean handkerchief or gauze pad. Place the pad over the socket and bite down firmly for 15 to 30 minutes. If bleeding does not stop the dentist should be contacted, repacking and suturing of the socket may be required

- Who to contact if they have excessive or prolonged post-operative bleeding

- To avoid taking non-steroidal anti-inflammatory drugs (NSAIDs) eg ibuprofen or aspirin for pain control immediately post-operatively. Paracetamol or its combination with codeine should be taken if pain control is needed

emphasis on preventive dentistry should also be highlighted to this patient group.

It is unsurprising that GDPs, with such a range of conflicting information having been given to them, should have such different views about the management of patients on warfarin. The summary of guidelines proposed in 2001 is summarised in Tables 2 and 3.

There is however, a clear need to address the concerns of GDPs in this area by prospective audits set in primary care, before their implementation. The Dental Practice Board could review the financial concern of GDPs in this respect by providing an additional payment on the grounds of increased complexity based on the Complexity component of the proposed Restorative Index of Treatment Need. ${ }^{30}$ There is also a need for prospective audits in relation to nerve blocks and some periodontal procedures in anticoagulated patients.

1. Hirsch J, Dalen J E, Deykin D, Poller L. Oral anticoagulants: mechanism of action, clinical effectiveness and optimal therapeutic range. Chest 1992; 102: suppl: 312S-326S.

2. Scully C, Cawson R A. Medical problems in dentistry. 3rd ed.pp 101. Oxford: Butterworth-Heinemann, 1993.

3. Devani P, Lavery K M, Howell C J T. Dental extractions in patients on warfarin: is alteration of anticoagulant regime necessary? Br J Oral Maxillofac Surg 1998; 36: 107-111.

4. Souto J C, Oliver A, Zuazu-Jausoro I et al. Oral surgery in anticoagulant patients without reducing the dose of oral anticoagulant: a prospective randomised study. J Oral Maxillofac Surg 1996; 54: 27-32.

5. Blinder D, Manor Y, Martinowitz U, Taicher S. Dental extractions in patients maintained on continued oral anticoagulant, comparison of local haemostatic modalities. Oral Surg Oral Med Oral Pathol Oral Radiol Endod 1999; 88: 137-140.
6. Gibbons A J, Evans L, Sayers M, Price G, Snooks H, Sugar A. Can warfarin be continued for dental extractions? Results of a randomised controlled trial. Abstract-Br J Oral Maxillofac Surg 1996; 54: 27-32.

7. Sindet-Pedersen S, Ramstrom G, Bernvil G et al. Haemostatic effect of tranexamic acid mouthwash in anticoagulant-treated patients undergoing oral surgery. NEng/J Med 1989; 320: 840-843.

8. Yeung C A. Letter to the Editor. BDA News 2001; $14: 35$.

9. Malden N J. Letter to the Editor. Br Dent J2003; 194: 65.

10. Dental Practitioners' Formulary. London: British Dental Association, British Medical Association and Royal Pharmaceutical Society of Great Britain, 20002002; D8.

11. Scully C, Cawson R A. Medical problems in dentistry. 4th ed.pp99. Oxford: Butterworth-Heinemann, 1998.

12. Surgical management of the primary care dental patient on warfarin. North West Medicines Information Centre. Ed Randall C: NHS:2001

13. Anon. Let's not wage war on warfarin! Br Dent J 2002; 193: 306.

14. Benoliel R et al. OralSurg Oral Med Oral Path 1986; 62: 149-151.

15. Scopp I W, Freric H. Dental extractions in patients undergoing anticoagulant therapy. Oral Surg Oral Med Oral Pathol 1958; 11: 470-74.

16. Waldrep A C, McKelvey L E. Oral Surgery for patients on anticoagulant therapy. J Oral Surg 1968; 26: 374-380.

17. Mulligan $R$, Weitzel $K G$. Pretreatment management of the patient receiving anticoagulant drugs. JADA 1988; 117: 479-483.

18. British National Formulary. London: British Medical Association and Royal Pharmaceutical Society of Great Britain, 2002; 116.

19. Wahl M J, Howell J. Altering anticoagulation therapy: a survey of physicians. JADA 1996; 127: 625-638.

20. Anon. Guidelines on oral anticoagulation: third edition. Br J Haematol 1998; 101: 374-387.

21. Bonow $\mathrm{R} O$ et al. ACC/AHA Guidelines for the Management of Patients With Valvular Heart Disease - A report of the American College of Cardiologyl American Heart Association Task Force on Practice Guidelines. J Heart Valve Disease 1998; 7: 672-707 and Circulation 1998; 18: 1949-1984.

22. Cannegeiter SC, Rosendaal F R, Briet E. Thromboembolic and bleeding complications in patients with mechanical heart valve prosthesis. Circulation 1994; 89: 635-641.

23. Cannegeiter S C, Rosendaal FR, Wintzen A R et al. Optimal oral anticoagulant therapy in patients with mechanical heart valves. N Engl J Med 1994; 333: 11-17.

24. Guidelines on the management of anticoagulation and antiplatelet therapy for endoscopic procedures. Gastrointestinal Endoscopy Online 1998; 48: 6: 1-5.

25. Webster $\mathrm{K}$, Wilde J. Management of anticoagulation in patients with prosthetic heart valves undergoing maxillofacial operations. Br J Oral Maxillofac Surg 2000; 38: 124-126.

26. Weibert R T. Oral anticoagulant therapy in patients undergoing dental surgery. Clin Pharmacol 1992; 11 : 857-864.

27. Madura J A, Rookstool M, Wease G. The management of patients on chronic coumarin therapy undergoing subsequent surgical procedures. Am Surg 1994; 60: 542-546

28. Bryan A J, Butchart E G. Prosthetic heart valves and anticoagulant management during non-cardiac surgery. Br JSurg 1995; 82: 577-578.

29. Kearon C, Hirsch J. Management of anticoagulation before and after elective surgery. N Eng/ J Med 1997; 336: 1506-1511.

30. Falcon FC etal. Developing an index of restorative dental treatment need. Br Dent J2001; 190: 479-486. 